\title{
Development of a Low-noise Front-end ASIC for CdTe Detectors
}

\author{
Tenyo Kawamura ${ }^{\mathrm{a}, \mathrm{b}, *}$, Tadashi Orita ${ }^{\mathrm{b}}$, Shin'ichiro Takeda ${ }^{\mathrm{b}}$, Shin Watanabe ${ }^{\mathrm{c}, \mathrm{b}}$, \\ Hirokazu Ikedac ${ }^{\mathrm{c}}$, Tadayuki Takahashi ${ }^{\mathrm{b}, \mathrm{a}}$ \\ ${ }^{a}$ Department of Physics, University of Tokyo, 7-3-1 Hongo Bunkyo, Tokyo 113-0033, Japan \\ ${ }^{b}$ Kavli Institute for the Physics and Mathematics of the Universe (WPI), University of Tokyo, 5-1-5 Kashiwanoha, Kashiwa, Chiba 277-8583, \\ Japan \\ ${ }^{c}$ Institute of Space and Astronautical Science, Japan Aerospace Exploration Agency (ISAS/JAXA), 3-1-1 Yoshinodai, Chuo-ku, Sagamihara, \\ Kanagawa 252-5210, Japan
}

\begin{abstract}
We present our latest ASIC, which is used for the readout of Cadmium Telluride double-sided strip detectors (CdTe DSDs) and high spectroscopic imaging. It is implemented in a $0.35 \mu \mathrm{m}$ CMOS technology (X-Fab XH035), consists of 64 readout channels and has a function that performs simultaneous $\mathrm{AD}$ conversion for each channel. The equivalent noise charge of $54.9 \mathrm{e}^{-} \pm 11.3 \mathrm{e}^{-}(\mathrm{rms})$ is measured without connecting the ASIC to any detectors. From the spectroscopy measurements using a CdTe single-sided strip detector, the energy resolution of $1.12 \mathrm{keV}$ (FWHM) is obtained at $13.9 \mathrm{keV}$, and photons within the energy from $6.4 \mathrm{keV}$ to $122.1 \mathrm{keV}$ are detected. Based on the experimental results, we propose a new low-noise readout architecture making use of a slew-rate limited mode at the shaper followed by a peak detector circuit.
\end{abstract}

Keywords: ASIC; Low-noise; X-ray; Gamma-ray; CdTe; Analog front-end

\section{Introduction}

Imaging spectroscopy of photons from $10 \mathrm{keV}$ to a few hundreds $\mathrm{keV}$ has a variety of applications in astronomy, medicine and industry. The wide use of this imaging technique has driven the development of imagers based on CdTe detectors, since they have high absorption efficiency comparable with that of $\mathrm{NaI}$ and $\mathrm{CsI}$, and the predominance of photoelectric absorption up to $\sim 250 \mathrm{keV}$ [1, 2, 3].

In the field of in-vivo molecular imaging, large detection area of $\sim 10 \mathrm{~cm}^{2}$ is required as well as the energy resolution of $\sim 1 \mathrm{keV}$ and spatial resolution of $\sim 100 \mu \mathrm{m}$ in order to image multiple radioisotopes[4]. A doublesided strip detector is a promising solution in terms of its small number of readout channels compared to a pixel detector. However large capacitance at the input of the signal processing circuit degrades the noise performance. It is therefore crucial to design a low-noise readout chip which operates under a large detector capacitance.

\footnotetext{
${ }^{*}$ Corresponding author

Email address: tenyo. kawamura@ipmu.jp (Tenyo Kawamura)
}

Preprint submitted to Journal of ${ }^{A T} T_{E} X$ Templates
We present our latest ASIC named KW04H64 which was designed for the readout of CdTe DSD having a detection area of $\sim 32 \mathrm{~mm} \times 32 \mathrm{~mm}$, a strip pitch of $250 \mu \mathrm{m}$, and a capacitance of $\sim 10 \mathrm{pF}$ for each channel. The ASIC has been modified from our previous versions (e.g. [5, 6]).

Section 2 describes the signal processing architecture of the ASIC and its predicted performance based on simulation results. Section 3 reports the first ASIC measurements. In Section 3.2 and 3.3 we discuss the measured noise performance and dynamic range of the ASIC when not connected to a detector. In Section 3.4. we evaluate the spectroscopic performance when the ASIC is connected to a CdTe and Silicon ( $\mathrm{Si}$ ) semiconductor detector, and in Section 4 we propose a new low-noise readout architecture.

\section{ASIC Description}

\subsection{Overview}

The KW04H64 ASIC is implemented in a CMOS $0.35 \mu \mathrm{m}$ technology (X-Fab XH035). The chip has 64 readout channels with self-trigger capability and it measures $7.12 \mathrm{~mm} \times 8.03 \mathrm{~mm}$. The dual power supply line 


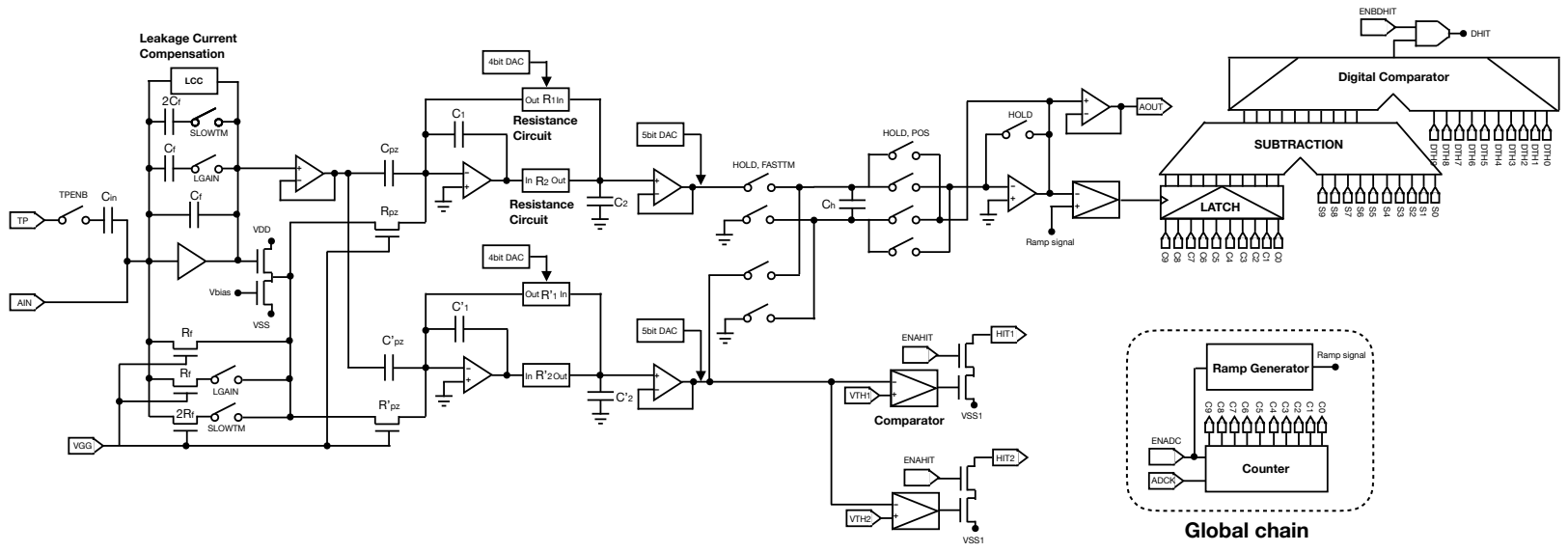

Figure 1: Schematic of the signal processing circuit implemented in each readout channel. Typical values of resistors and capacitors are $R_{\mathrm{f}}=5 \mathrm{G} \Omega$, $C_{\mathrm{f}}=0.032 \mathrm{pF}, R_{\mathrm{pz}}=50 \mathrm{M} \Omega, C_{\mathrm{pz}}=3.2 \mathrm{pF}, R_{1}=R_{2}=8 \mathrm{M} \Omega, C_{1}=0.4 \mathrm{pF}, C_{2}=0.1 \mathrm{pF}, R_{1}^{\prime}=R_{2}^{\prime}=0.8 \mathrm{M} \Omega, C_{1}^{\prime}=0.4 \mathrm{pF}, C_{2}^{\prime}=0.1 \mathrm{pF}$ and $C_{\mathrm{h}}^{\prime}=0.8 \mathrm{pF}$. Note that the ramp signal generator and the counter used in the Wilkinson ADC are implemented in another block in the ASIC whose outputs are provided to each channel.

Table 1: Main characteristics of the ASIC KW04H64.

\begin{tabular}{ll}
\hline Parameter & Value \\
\hline Fabrication process & X-Fab XH035 \\
Chip size & $7.12 \mathrm{~mm} \times 8.03 \mathrm{~mm}$ \\
Number of channels & 64 \\
Power rail & $\pm 1.65 \mathrm{~V}$ \\
Polarity & Both \\
Gain & $170 \mathrm{mV} / \mathrm{fC}$ \\
Dynamic range & $\sim 6.0 \mathrm{fC}$ \\
ENC & $58.4 \mathrm{e}^{-}+12.7 \mathrm{e}^{-} / \mathrm{pF}$ (Fast shaper) \\
& $33.0 \mathrm{e}^{-}+5.2 \mathrm{e}^{-} / \mathrm{pF}$ (Slow shaper) \\
Peaking time & $\sim 0.5 \mu \mathrm{s}$ (Fast shaper) \\
Power consumption & $\sim 2.0 \mu \mathrm{s}$ (Slow shaper) \\
\hline
\end{tabular}

of $\pm 1.65 \mathrm{~V}$ has been adopted to process the signal in either polarity, which is required for DSDs. The main characteristics of the ASIC are summarized in Table 1

Figure 1 shows the signal processing architecture of the ASIC. This architecture is the same as the previous KW04D64 chip version[7], with minor modifications concerning the circuit gain and includes additional functions. It has a dual path topology after the charge sensitive amplifier (CSA). As the names suggest, the "timing branch" is dedicated to measuring time, and the "energy branch" is dedicated to measuring energy. The timing branch has a pole-zero cancellation (PZC), a 2nd-order low-pass filter, and two separate comparators which generate hit signals. The energy branch has same architecture as that of the timing branch before comparators, followed by a sample-and-hold circuit and a Wilkinson ADC which enables the AD conversion for all the channels to happen simultaneously. The PZC and the lowpass filter effectively work as the CR-RC shaper, which is called the "fast shaper" and "slow shaper" for the timing and energy branches and results from simulations give peaking times of $\sim 0.5 \mu \mathrm{s}$ and $\sim 2.0 \mu \mathrm{s}$, respectively. The 5-bit and 4-bit DACs employed for the baseline adjustment have a pair of output ports. The current supplied from one port of the DAC is designed to be recovered by the other port, which avoids any interference to adjacent circuits such as the buffer amplifier. The sample-and-hold circuit is triggered by an external control block at a given time after a hit signal is generated. The fast shaper signal from the timing branch can be selected as an input of the Wilkinson ADC in the energy branch. This is beneficial for the performance measurement and the baseline adjustment of the fast shaper.

The signals are read out after the AD conversion at the Wilkinson ADCs for each event. The ASIC has a sparse readout mode as well as a full readout mode. In the sparse readout mode, only those channels carrying the signal data are read out in order to reduce the output data size. Whether each channel carries the signal is judged by either the state of the hit signal (HIT1 in Figure1 or the value of ADC subtracted by low frequency common mode noise[8]. 


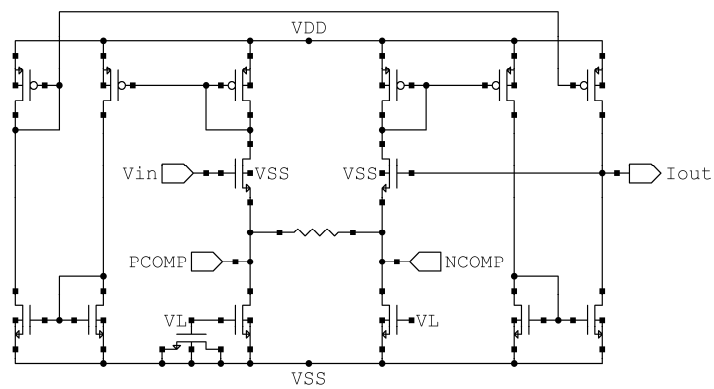

Figure 2: Schematic of the resistance circuit implemented in the shaper. Connected to a current DAC, PCOMP and NCOMP can be used to calibrate a baseline coarsely.

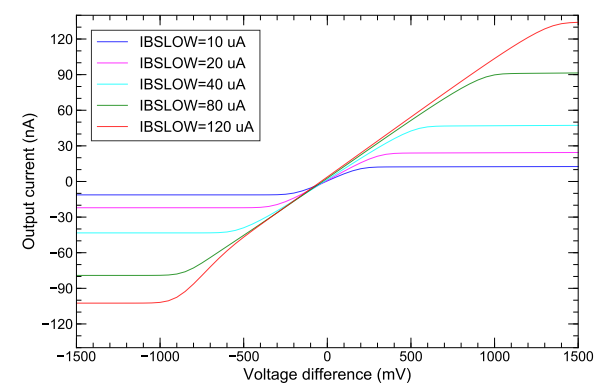

Figure 3: DC performance of the resistance circuit at the slow shaper predicted by simulations.

\subsection{Details of the analog signal processing}

The CSA employs a folded cascode scheme with a PMOS input transistor having $W / L=1440 \mu \mathrm{m} / 1.2 \mu \mathrm{m}$ providing a transconductance of $\sim 3 \mathrm{mS}$ with a drain current of $\sim 160 \mu \mathrm{A}$. The drain current can be changed by an externally located potentiometer. In this paper, the feedback capacitance at the CSA is fixed at $0.032 \mathrm{pF}$, although, depending on the photon energy, it can be set to $0.032 \mathrm{pF}, 0.064 \mathrm{pF}, 0.096 \mathrm{pF}$, and $0.128 \mathrm{pF}$. The feedback resistor is implemented using an NMOS. Its resistance is controlled by the gate voltage VGG, which is generated by the internally located DAC. Although it was switched off in this paper, a leakage current compensation circuit is implemented which can deal with up to $\sim 1 \mathrm{nA}$ for either polarity (see [6, 9] for details).

At the shapers, the transconductors play an important role in the circuit performance. They are referred to as high resistance circuits having $\sim 8 \mathrm{M} \Omega$ for the slow shaper and $\sim 0.8 \mathrm{M} \Omega$ for the fast shaper. The resistance circuits used in both the fast shaper and the slow shaper are implemented using the same architecture shown in Figure 2, where two-stage current mirror scales down the current flowing into the passive resistor. The bias current at the resistance circuits is controlled externally by changing the reference current in a similar manner to the drain current of the input PMOS at the CSA. The reference current for the resistance circuits at the slow shaper is a key parameter in the paper, and is referred to hereafter as IBSLOW. Note that the two resistance circuits at each shaper are biased in common to make the poles degenerated, i.e., to keep track of the critical damping condition.

The reference current determines the amount of output current. The maximum output current $I_{\mathrm{SR}}$ from the resistance circuit at the slow shaper is $I_{\mathrm{SR}} \sim$ (1/1000)IBSLOW. Limiting the output current also limits the voltage difference under which the resistance circuit behaves as a linear resistor. The maximum voltage difference $V_{\text {res,max }}$ is expressed as $V_{\text {res,max }}=R_{\text {res }} I_{\mathrm{SR}}$ where $R_{\text {res }}$ is its resistance, i.e., $\sim 8 \mathrm{M} \Omega$ for the slow shaper. When the voltage difference is larger than $V_{\text {res,max }}$, the resistance circuit switches to the slew-rate limited mode, where it can only source or sink a maximum amount of current given by $I_{\mathrm{SR}}$. Figure 3 shows the DC performance of the resistance circuit at the slow shaper predicted by simulations where the output voltage is fixed at $0 \mathrm{~V}$, meaning the voltage difference described in the figure represents the input voltage. It has been implied that the slew-rate limited mode contributes to better noise performance[10, 11].

According to simulations, the maximum signal to be processed is expected to be $\sim 6 \mathrm{fC}$ with the gain of $\sim 170 \mathrm{mV} / \mathrm{fC}$, while the noise performance is expected to be ENC $=58.4 \mathrm{e}^{-}+12.7 \mathrm{e}^{-} / \mathrm{pF}$ and $\mathrm{ENC}=$ $33.0 \mathrm{e}^{-}+5.2 \mathrm{e}^{-} / \mathrm{pF}$ for the fast shaper and the slow shaper respectively.

\section{Experimental Results}

\subsection{Experimental Setup}

The ASIC performance was evaluated both without a detector and with Si and CdTe detectors. In the setup without a detector, an ASIC was placed in a QFP ceramic package on a test board and test pulses generated internally were used as the input signals. The analog input pads of 56 readout channels are floated, while for 8 channels each pad is connected to a pin on the test board. Due to the difference between these two types of configurations, 56 channels were used for the performance evaluation. In the setup with a detector, bare chips were connected to single-sided strip detectors characterized in Tables 2 and 3, where the input signals to ASICs have positive polarity. The interface with a computer was established using the SpaceWire 
Table 2: Main characteristics of the CdTe detector.

\begin{tabular}{ll}
\hline Parameter & Value \\
\hline Manufacturer & ACRORADO \\
Type & Schottky CdTe diode \\
Size & $4 \mathrm{~mm} \times 4 \mathrm{~mm}$ \\
Thickness & $1 \mathrm{~mm}$ \\
& 16 strip electrodes \\
Pt side & $250 \mu \mathrm{m}$ pitch \\
In side & 1 plain electrode \\
\hline
\end{tabular}

Table 3: Main characteristics of the Si detector.

\begin{tabular}{ll}
\hline Parameter & Value \\
\hline Manufacturer & Hamamatsu Photonics \\
Type & PN Si diode \\
Size & $12.8 \mathrm{~mm} \times 12.8 \mathrm{~mm}$ \\
Thickness & $300 \mu \mathrm{m}$ \\
P side & 32 strip electrodes \\
& $400 \mu \mathrm{m}$ pitch \\
\hline
\end{tabular}

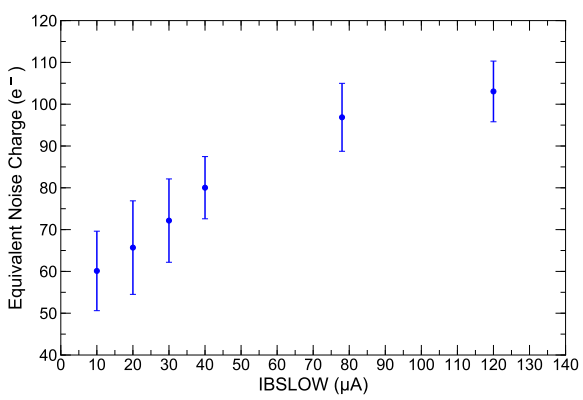

Figure 4: Noise performance at the slow shaper with respect to IBSLOW. The amplitude of the test pulse is $-0.93 \mathrm{fC}$. The error bars represent one-sigma among the 56 channels.

DIO2[12] which contains a reconfigurable FPGA. The dedicated software was written for the ASIC operation on the computer.

\subsection{Noise Performance}

Figure 4 shows the noise performance with respect to the reference current for the resistance circuit at the slow shaper. It was measured by injecting 1000 test pulses and then examining distributions of ADC values for 56 channels. The amplitude of the test pulses is $-0.93 \mathrm{fC}$ and the hold timing with respect to the hit timing was kept as $3.7 \mu$ s for IBSLOW $\geq 40 \mu \mathrm{A}$, while for IBSLOW $=30,20$ and $10 \mu \mathrm{A}$ it is set at 4.0,4.5 and $5.2 \mu$ s because of the slew-rate limited mode in the slow shaper, respectively (see Figure 6). From the figure, the noise performance is improved more sharply as IBSLOW becomes smaller for IBSLOW $\lesssim 40 \mu \mathrm{A}$ than

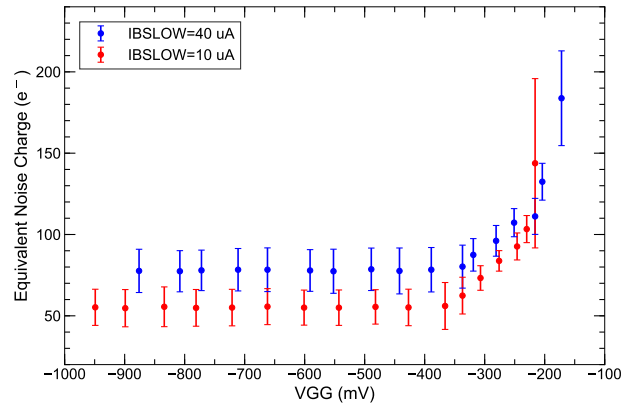

Figure 5: Noise performance at the slow shaper with respect to VGG. The amplitude of the test pulse is $-0.93 \mathrm{fC}$. The error bars represent one-sigma among the 56 channels.

for IBSLOW $\gtrsim 40 \mu \mathrm{A}$. We conjecture that the noise reduction for IBSLOW $\lesssim 40 \mu \mathrm{A}$ is related to the slew-rate limited mode of the resistance circuit.

Figure 5 shows the ENC versus the gate voltage of the NMOS used as the feedback resistor at the CSA when IBSLOW $=10$ and $40 \mu \mathrm{A}$. The feedback resistance increases along with an increase in the absolute value of the VGG, which leads to better noise performance, but at the expense of longer decay constants. In Figure 5 , the ENC settles down when the noise from the NMOS becomes negligible compared to that from other components. The best noise performance with the smallest $\mathrm{ENC}=54.9 \mathrm{e}^{-} \pm 11.3 \mathrm{e}^{-}(\mathrm{rms})$ is achieved at the lowest value of IBSLOW $=10 \mu \mathrm{A}$.

\subsection{Dynamic Range Performance}

Figure 6 shows the waveforms at the slow shaper for various input charge under various IBSLOW. The waveforms extend for smaller IBSLOW. This peak distortion is due to the behavior of the resistance circuit at the slow shaper. For smaller IBSLOW, the resistance circuit is apt to work in the slew-rate limited mode and can provide small current, which limits the speed of the voltage at the output of the slow shaper. It is also observed that negative pulses make the waveform more distorted than that of positive pulses. This asymmetry is also caused by the resistance circuit where the input NMOS shows a different behavior as a response to positive and negative inputs.

Figure 7 shows the relation between the input charge and the ADC value representing the amplitude of the sample-and-hold voltage from the slow shaper for various IBSLOW. The results from a typical channel are plotted. The hold timing with respect to the hit timing was $3.7 \mu$ s for IBSLOW $\geq 40 \mu \mathrm{A}$ and $5.2 \mu \mathrm{s}$ for IBSLOW $=10 \mu \mathrm{A}$. The gain dispersion was $3-8 \%$ in 

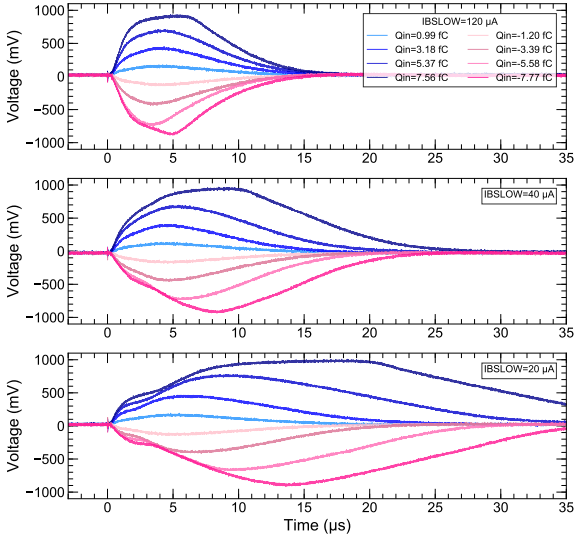

Figure 6: Waveforms at the output of the slow shaper for various input charge under various IBSLOW.

one-sigma among the 56 channels. The dynamic range was found to be $\sim 6.5 \mathrm{fC}$ at IBSLOW $=120 \mu \mathrm{A}$ and became smaller following the decrease in IBSLOW, reaching $\sim 2.0 \mathrm{fC}$ at IBSLOW $=10 \mu \mathrm{A}$. This trend can be well explained by the behavior of the resistance circuit at the slow shaper and the sample-and-hold circuit. Since in the sample-and-hold circuit the voltage is sampled at a given time after the hit signal is issued, it is impossible to correctly capture the peak if it moves with the signal charge.

\subsection{Spectroscopic Performance}

The spectra in Figure 8 were acquired using the CdTe detector with ${ }^{241} \mathrm{Am},{ }^{57} \mathrm{Co}$ and ${ }^{133} \mathrm{Ba}$ sources, where the detector was biased at $1000 \mathrm{~V}$ and cooled at $-20^{\circ} \mathrm{C}$. The slow shaper was selected as the input of the ADC, and the reference current was set at IBSLOW $=40 \mu \mathrm{A}$. We confirmed the detection of photons whose energy ranges from $6.4 \mathrm{keV}$ to $122.1 \mathrm{keV}$. The energy resolution at $13.9 \mathrm{keV}$ (FWHM) was found to be $1.12 \mathrm{keV}$. We also found that energy resolution becomes worse as the peak energy increases, which we will investigate further.

Figure 9 shows the spectrum acquired using an ${ }^{241} \mathrm{Am}$ source, where the $\mathrm{Si}$ detector was biased at $80 \mathrm{~V}$ and cooled at $-10^{\circ} \mathrm{C}$. The slow shaper was selected as the input of the ADC, and the reference current was set at IBSLOW $=40 \mu \mathrm{A}$. The energy resolution was found to be $1.2 \mathrm{keV}$ at $59.5 \mathrm{keV}$.

\section{New low-noise readout architecture}

According to the results reported in Section 3.2 and 3.3. the slew-rate limited mode offers better noise performance with some penalty in the dynamic range as

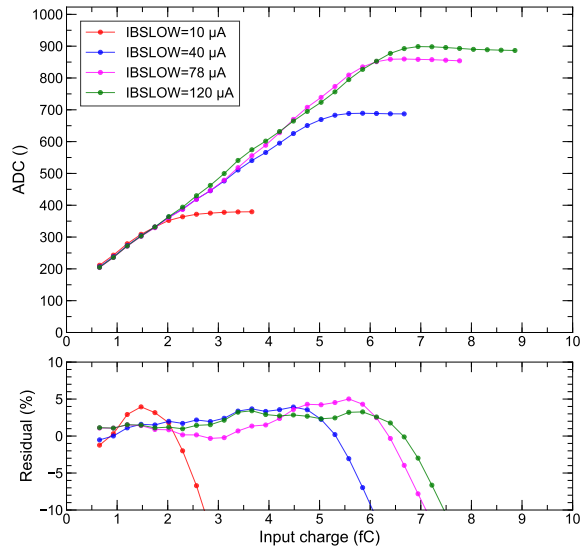

Figure 7: The upper plot shows ADC values with the input from the slow shaper with respect to the input charge of a channel for different IBSLOW values. The data are fitted over the dynamic range for each IBSLOW value. Residuals are defined as the difference of the measurement divided by the saturated ADC value, and are shown in the lower plot. The signals with negative polarity are used.

long as the sample-and-hold circuit is employed. Figure 10 shows the peaking time and the peak amplitude with respect to the negative input charge. The peaking time gets longer as the input charge increases when the slow shaper works in the slew-rate limited mode, while the peak voltage maintains the linearity regardless of the mode for the input charge up to and above $8 \mathrm{fC}$. This implies that the combination of the slew-rate limited mode and the peak detector circuit should satisfy both the noise performance and the wide dynamic range.

On the other hand, it should be noted that there exist some reservations to employ the peak detector circuit with the slew-rate limited mode. This architecture makes it hard to detect the common mode noise. Care must be taken so that the AD conversion starts after the voltage reaches its peak for the largest signal to be targeted. In addition, the peaking time reflects noise performance. These matters must be taken into account in employing this architecture.

\section{Summary}

The KW04H64 ASIC has been designed for the readout of CdTe DSD allowing for high spectroscopic imaging. Evaluating its performance experimentally, the low noise performance, ENC of $54.9 \mathrm{e}^{-} \pm 11.3 \mathrm{e}^{-}$(rms), has been demonstrated without any detector. From the evaluation of the spectroscopic performance using the CdTe single-sided detector, the ASIC demonstrated the high 

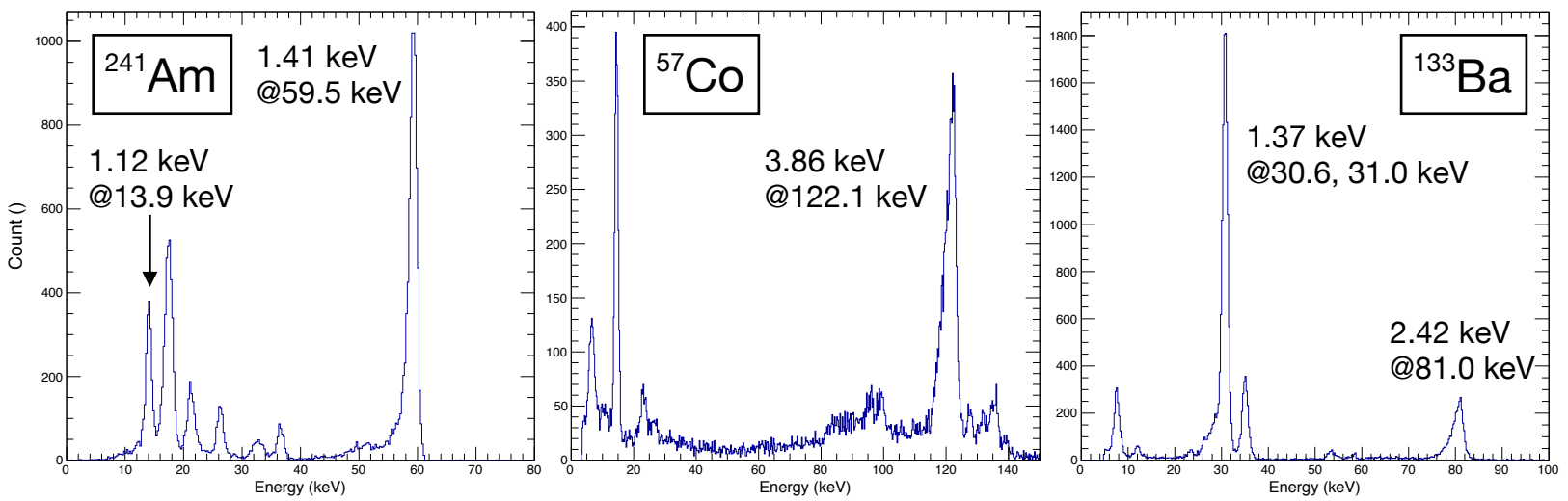

Figure 8: Energy spectra acquired with various sources from one channel where only single hit events were extractred.

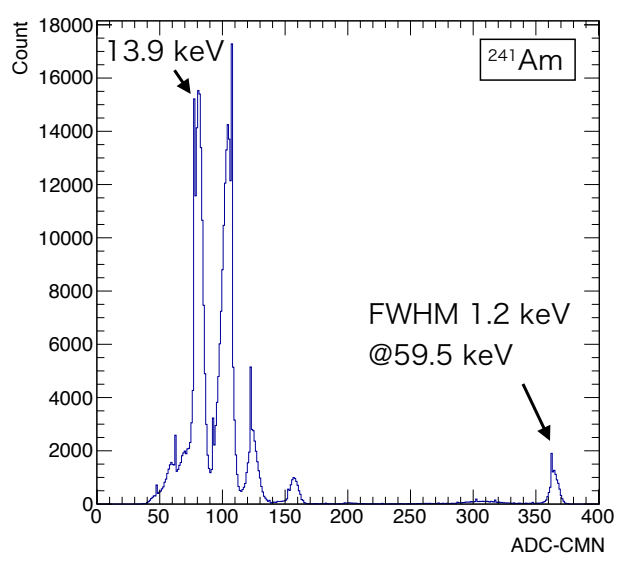

Figure 9: Energy spectrum acquired using an ${ }^{241}$ Am source from one channel where only single hit events were extractred. The horizontal axis represents the value of ADC subtracted by common mode noise.

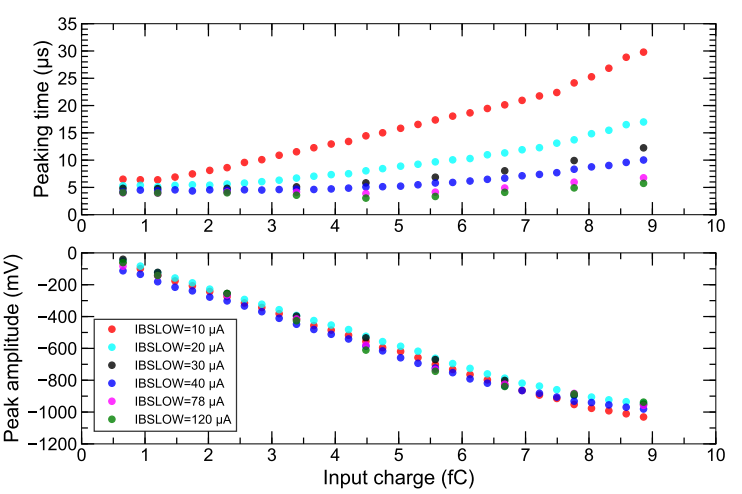

Figure 10: Peaking time and amplitude with respect to the test pulse negative input charge for different IBSLOW values. The peaking time is defined as the time when the waveform reaches its minimum value. energy resolution of $1.12 \mathrm{keV}$ for the energy at $13.9 \mathrm{keV}$ and the capability of detecting photons within the energy from $6.4 \mathrm{keV}$ to $122.1 \mathrm{keV}$. Investigating the circuit behavior under the slew-rate limited mode of the slow shaper, we propose a new readout architecture incorporating a peak detector circuit to achieve a low noise readout without sacrificing the dynamic range. The confirmation of the readout architecture and the performance evaluation of the CdTe DSD will follow.

\section{Acknowledgement}

This work was supported by JSPS KAKENHI grant number $18 \mathrm{H} 05463$. The authors thank P. Caradonna for his critical reading of the manuscript.

\section{References}

[1] T. Takahashi, et al., IEEE Trans. Nucl. Sci. 48 (2001) 950 - 959.

[2] L. Jones, et al., Nucl. Instrum. Meth. A 604 (2009).

[3] A. Meuris, et al., IEEE Trans. Nucl. Sci. 56 (2009) 1835 - 1841.

[4] S. Takeda, et al., Nucl. Instrum. Meth. A 912 (2018) 57 - 60.

[5] H. Ikeda, Nucl. Instrum. Meth. A 569 (2006) $98-101$.

[6] T. Kishishita, et al., IEEE Trans. Nuc. Sci. 57 (2010) 29712977.

[7] A. Harayama, et al., Nucl. Instrum. Meth. A 765 (2014) 223 226.

[8] H. Ikeda, et al., Nucl. Instrum. Meth. A 389 (1997) $454-462$.

[9] F. Krummenacher, Nucl. Instrum. Meth. A 305 (1991) 527-532.

[10] G. Sato, et al., IEEE Trans. Nucl. Sci. Symposium Conference Record (2009).

[11] G. Sato, et al., IEEE Trans. Nucl. Sci. 58 (2011) 1370 - 1375.

[12] Shimafuji Electric Incorporated (2020-05-11 accessed). URL: http://www.shimafuji.co.jp/en/products/508 